\title{
Waveguide Mode Filter Fabricated Using Laser-Induced Forward Transfer
}

\author{
K.S.Kaur, A.Z.Subramanian, D.P.Banks, M.Feinaeugle, C.Y.J.Ying, C.L.Sones, S.Mailis, R.W.Eason \\ Optoelectronics Research Centre, University of Southampton, Southampton, SO17 1BJ, UK \\ kak@orc.soton.ac.uk
}

\begin{abstract}
Titanium in-diffused lithium niobate index-tapered waveguides have been fabricated using laser-induced forward transfer technique for mode-filtering applications. Details of their fabrication, losses and transmission characterization are presented.

OCIS codes: (140.3390) Laser materials processing; (140.7090) Ultrafast lasers; (220.4000) Microstructure fabrication; Laser-Induced Forward Transfer; (230.7370) Waveguides.
\end{abstract}

\section{Introduction}

In this paper we report the fabrication of titanium (Ti) in-diffused lithium niobate (LN) index-tapered waveguides using a method based on laser-induced forward transfer (LIFT) [1], and thermal diffusion techniques [2]. This approach offers the flexibility, less stringent experimental conditions and rapid prototyping associated with LIFT, and the large refractive index change, low optical loss and stability of waveguides produced with the thermal diffusion method.

\section{Experiments and results}

The fabrication of tapered waveguides was a two-step process. First, segmented lines of Ti metal were printed on top of LN substrates using the LIFT technique. The LIFT samples were prepared by depositing thin films $(\sim 150$ $\mathrm{nm}$ ) of $\mathrm{Ti}$ (the donor) on top of transparent glass substrates (the carrier) by e-beam evaporation. Demagnified femtosecond (fs) pulses from a Ti: sapphire laser $(800 \mathrm{~nm}, 150 \mathrm{fs})$ were then imaged at the carrier-donor interface and $\mathrm{Ti}$ dots of diameter $\sim 10-12 \mu \mathrm{m}$ (comparable to the incident laser spot size) were printed onto a congruent undoped $\mathrm{z}$-cut $\mathrm{LN}$ substrate (the receiver) on the $-\mathrm{z}$ face along the crystallographic $\mathrm{y}$-direction. The laser fluence threshold value for transfer of Ti dots was $\sim 0.4 \mathrm{~J} / \mathrm{cm}^{2}$. All experiments were performed under a background pressure of $10^{-1}$ mbar and the laser was operated at a repetition rate of $250 \mathrm{~Hz}$. After printing, the metal lines were in-diffused into the $\mathrm{LN}$ substrates by heating at $1050^{\circ} \mathrm{C}$ in an oxygen atmosphere for 10 hours.

The donor-receiver assembly was mounted on a computer-controlled 3D translation stage to achieve relative movement with respect to the incoming laser pulses. The separation between two consecutive Ti dots was controlled by varying the speed of the translation stages. To realize index tapered waveguides, different samples were prepared by varying the writing speeds across the segmented Ti lines at a constant acceleration. The consequence of the constant acceleration is that the segment separation increases linearly with increasing scan speed thereby decreasing the average amount of deposited $\mathrm{Ti}$ and subsequently reducing the average $\mathrm{Ti}$ concentration in the crystal, after diffusion. The variation of the Ti concentration corresponds to a variation of the average refractive index along the length of the waveguide. The mode confinement therefore decreases with increasing speed and the waveguide modal behavior changes from multi-mode to single-mode. By increasing the segment separation and hence the average refractive index contrast along the length of the waveguide, a mode filter that allowed only the fundamental mode to propagate was realized. In the present set of experiments three different values of constant acceleration $0.3,0.4$ and $0.5 \mathrm{~mm} / \mathrm{s}^{2}$ were used for fabricating the tapered waveguides. The initial velocity was kept constant at $2.5 \mathrm{~mm} / \mathrm{s}$ for all the tapers. Waveguides with a constant velocity of $2.5 \mathrm{~mm} / \mathrm{s}$ that corresponds to a segment separation (distance between the centres of adjacent segments) of $10 \mu \mathrm{m}$ were also written for comparison. All the samples were then end polished for optical characterisation and loss measurements.

The waveguide losses were measured using three different techniques namely Fabry-Perot (FP); fibre mismatch and mode mismatch. All these losses were measured at $1550 \mathrm{~nm}$ and all samples were $1.6 \mathrm{~cm}$ in length. The average propagation loss obtained for the tapers was $3.8 \pm 0.8 \mathrm{~dB} / \mathrm{cm}$. The variation in the velocity along the waveguide length leads to higher values of losses in tapers than the constant velocity waveguides as reported in our earlier work [3]. For optical characterization of the waveguides light from a tunable fiberised laser (1500-1600 nm) was coupled into the waveguides using an objective lens $(40 \mathrm{X})$. The output was collected using another objective lens $(40 \mathrm{X})$ and the mode profiles at the output of the waveguides were observed using an IR camera. The optical characterization was performed at $1550 \mathrm{~nm}$. Figure 1(a) shows a higher order mode profile obtained from a segmented waveguide fabricated with a constant velocity of $2.5 \mathrm{~mm} / \mathrm{s}$. Figure 1 (b), (c) and (d) show the optical mode profiles of index- tapered waveguides written with constant acceleration of $0.3,0.4$ and $0.5 \mathrm{~mm} / \mathrm{s}^{2}$ respectively 
with an initial velocity of $2.5 \mathrm{~mm} / \mathrm{s}$. The dense end of the taper can support higher order modes as shown in Fig 1(a) however, transmission through the waveguide with a variation of the refractive index along its length results in a mode filtering action as it is only the fundamental mode that will be finally transmitted. This is a consequence of the fact that as the separation between the printed dots increases the average effective index decreases accordingly leading to cut-off of the higher order modes leaving only the fundamental mode propagating. Another important feature to be noticed is that as the writing speed/acceleration was increased the mode size increased as well. This is because the decrease in the index contrast with increasing segment separation leads to a broader and less tightly confined mode. This effect can be utilized for the fabrication of mode size converters.

Tapered waveguides

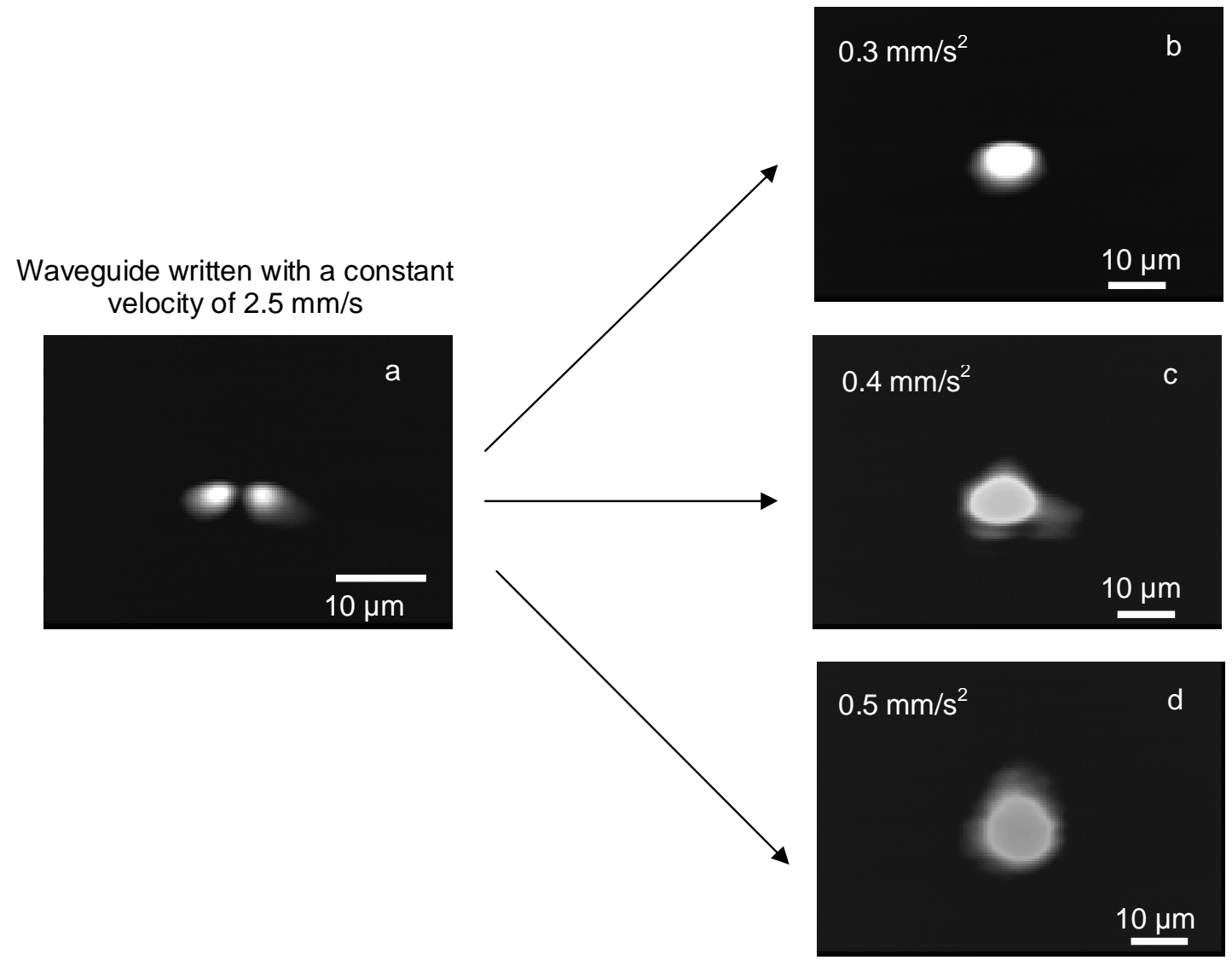

Fig. 1. (a) Shows the near-field mode profiles captured from a waveguide written with a constant velocity of $2.5 \mathrm{~mm} / \mathrm{s}$ and 1 (b-d) shows the mode profiles of tapered waveguides written at accelerations of $0.3,0.4$ and $0.5 \mathrm{~mm} / \mathrm{s}^{2}$ respectively.

\section{Conclusions}

Index-tapered waveguides fabricated using a simple yet powerful technique based on the established LIFT process and in-diffusion methods are reported for mode filtering applications. The propagation losses of the tapers were measured using three different techniques and the average losses were $\sim 3.8 \pm 0.8 \mathrm{~dB} / \mathrm{cm}$ at $1550 \mathrm{~nm}$. The mode profile pictures captured confirmed the mode filtering action.

\section{References}

[1] J. Bohandy, B.F. Kim, F.J. Adrian, “Metal deposition from a supported metal film using an excimer laser,”J. Appl. Phys. 60, 1538 (1986). [2] R.C. Alferness and L.L. Buhl, "Efficient waveguide electro-optic TE $\leftrightarrow$ TM mode converter/wavelength filter," Appl. Phys. Lett. 36, 513 (1980).

[3] C.L. Sones , K.S. Kaur, P. Ganguly, D.P. Banks, Y.J. Ying, R.W. Eason, S. Mailis, “ Laser-induced-forward-transfer: a rapid prototyping tool for fabrication of photonic devices," Appl. Phys. A. 101,333-338 (2010). 